\title{
FURTHER OBSERVATIONS ON PINK DISEASE
}

\author{
BY \\ J. VERNON BRAITHWAITE, M.D., M.R.C.P. \\ (From the Children's Hospital, Leicester Royal Infirmary.)
}

In a previous communication ${ }^{1}$ the suggestion was made that pink disease is an abnormal reaction to daylight in an infected child. The chief evidence given in favour of this was as follows:-

The condition usually occurs after an acute infection of the respiratory tract; there is' a marked seasonal incidence in the spring and summer (in this country) only four out of twenty-seven cases being admitted during the winter; twenty of the twenty-seven patients came from the country or suburbs; the disease was first described in Australia, and is prevalent in sunny countries; and, finally, protecting the children from daylight by keeping them in a room with windows of ruby glass caused a rapid improvement in their condition.

Further experience has confirmed this view. In 1933 and 1934 a further twenty-two patients suffering from this complaint were admitted to the Leicester Children's Hospital, making a total of forty-nine cases from the district since 1923. Of these forty-nine patients only eight were admitted between the months of November and February inclusive, while forty-one were admitted in the period from March to October. It is thus seen that the disease is one occurring in the time of long days and short nights, while during the coldest and darkest days it almost dies out. At the height of summer, however, the curve falls slightly, the greatest incidence being in April and August.

CHART I.

Seasonal incidence.

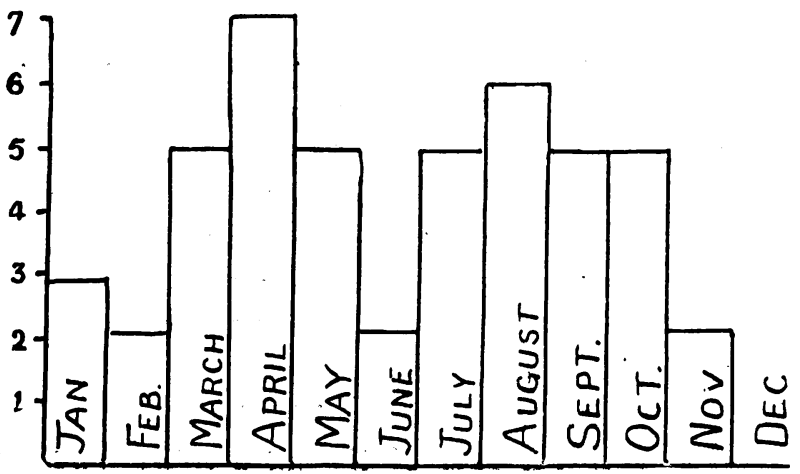


The seasonal incidence, therefore, supports the hypothesis that the disease is in some way related to daylight. The ultra-violet rays, the rays of the visible spectrum, or the heat rays could conceivably be responsible. In this connection it is interesting to note that among the patients admitted in 1933, in which the summer was unusually hot, the death rate was high. Of ten patients admitted six died, and this was in spite of their being placed in a red windowed room, a manoeuvre which had previously apparently been successful. The case of one of these patients in particular is instructive and tragic.

He was a boy of nine months, and was admitted in May. At the time of his admission the ' red room' was occupied, and he was kept in the ward until it should be empty. On the morning of the second day the sun came round to the window above his cot, and owing to the time being that when the night and day staff changed over, this was unnoticed for half an hour. The child became very ill with a high temperature and a raised pulse and respiration rate and died the same evening.

It will be remembered that in the previous paper already referred to a patient with pink disease was deliberately placed in the sunlight and showed toxic symptoms within an hour, but recovered on being taken back indoors.

\section{The effect of sunlight on blood.}

In 1917 Clare $^{2}$, investigating the toxic effects of destroyed red blood cells, found that sunlight rapidly destroyed washed red corpuscles suspended in normal saline. Moreover he found that these destroyed cells possessed toxic properties when injected subcutaneously. This work was done in India, and in view of the association of pink disease with hot and sunny weather it was decided to perform similar experiments in this country. Blood from various individuals was collected with aseptic precautions from a finger prick in amounts of 0.1 c.c. and was suspended in 2 c.c. normal saline ( $\mathrm{pH} \mathrm{7-8)}$ in test tubes of about 5 c.c. capacity. The blood was mixed with the saline by placing a finger covered with a sterile rubber finger stall on the open end of the tube' and inverting. (Test cultures from these tubes proved that they were sterile.) The tube was then plugged with sterile cotton wool and was placed in the direct sunlight for three hours. It was then kept in the dark until the following morning (about eighteen hours) and readings for haemolysis were taken.

Blood was taken from thirty-one children between the ages of four months and nine years, suffering from various complaints, including four cases of pink disease, from three normal adults, and from four adult patients. It was found that in thirty out of thirty-eight cases complete haemolysis had occurred. The eight specimens which showed no haemolysis were all from patients with some form of anaemia. The blood from the normal adults haemolyzed as readily as the others, while that from the patients with pink disease showed no difference from the rest either in the rapidity or the extent of the haemolysis.

This destructive action of sunlight might be due to any of the three factors mentioned above-the rays of the ultra-voilet end of the solar 
spectrum, some of which may pass through glass and are known to be haemolytic; the rays of the visible spectrum; or the heat rays from the infra-red portion. It was decided to submit specimens of blood to the influence of each of these to determine which was the haemolyzing agent.

The effect of irradiating blood in vitro by ultra-violet light was investigated by Mr. C. J. Bond. Suspensions of $0 \cdot 1$ c.c. of blood in 2 c.c. of normal saline were used. Two suspensions were made in glass tubes similar to those used in the previous experiment, and two were made in quartz tubes of similar size. All four tubes were then subjected to irradiation from a mercury-vapour lamp. Complete haemolysis occurred in the quartz tubes, but none in the glass tubes. From this it was concluded that although ultra-violet light is a powerful haemolytic agent, the haemolysis obtained by exposing blood in glass tubes to sunlight was not due to this part of the solar spectrum.

The effect of white light on blood was investigated by adapting a microphotographic apparatus. An aluminium plate with a slit in it was placed in front of a two kilowatt lamp, and the rays coming through the slit were concentrated by means of a powerful convex lens, mounted on the optical bench, on to the glass tubes containing the blood suspended in saline. Three specimens of blood from normal adults and two from patients with pink disease were thus irradiated for six hours each. In no case was any haemolysis produced.

The effect of heat as a haemolytic agent is well known. To complete this investigation, however, suspensions of blood in saline were immersed in water baths at temperatures of $40^{\circ} \mathrm{C}, 52^{\circ} \mathrm{C}$, , and $55^{\circ} \mathrm{C}$. Three specimens, one from a normal adult, one from a child convalescent from pneumonia, and one from a patient with pink disease, were immersed in the water bath at $40^{\circ} \mathrm{C}$. for three hours, but no haemolysis occurred. Seven specimens (three from patients with pink disease) were immersed at a temperature of $52^{\circ} \mathrm{C}$. Haemolysis occurred in all cases in time varying from one-and-a-half to three hours. Five specimens (two from patients with pink disease) were immersed at a temperature of $55^{\circ} \mathrm{C}$. All showed haemolysis, four of them being haemolyzed in one-and-a-half hours, and one from a normal adult in two hours.

It appears therefore that the haemolysis produced by exposing blood suspended in saline in vitro to direct sunlight is due to the effect of heat.

\section{Skin sensitivity to blood haemolyzed by heat.}

The above results, taken in conjunction with Clare's work already referred to, suggested the possibility that pink disease is of the nature of an allergic reaction to heated blood. Intradermal injection of heated blood, however, failed to confirm this: $0 \cdot 1$ c.c. of citrated blood, haemolyzed by heat, was injected intradermally into the forearm of a patient with pink disease, and a similar amount of unheated citrated blood was injected into the skin of the other forearm. In fifteen minutes a small urticarial wheal 
had formed at the site of each injection, there being no marked difference in the size of wheals. As a control similar tests were made on a patient suffering from Fröhlich's syndrome with identical results.

The effect of subcutaneous injection of heated blood.

Citrated blood, haemolyzed by heat, was injected in increasing doses into the subcutaneous tissues of a patient suffering from pink disease, and at the same time similar injections were given to a patient with mild nutritional anaemia as a control. The amounts injected were $2,4,10$, and 12 c.c. at weekly intervals. The patient with nutritional anaemia showed no reaction. The patient with pink disease showed no reaction until the dose of 12 c.c. was reached. About eighteen hours later the pulse rate rose from 124 to 160 per minute. In a further twelve hours the temperature rose from the normal level to $103.8^{\circ} \mathrm{F}$., and the respiration rate to over 90 per minute. There was collapse, sweating, and an aggravation of the swelling and pinkness of the hands and feet. No abnormal physical signs were detected in the lungs or elsewhere. This condition persisted for forty-eight hours, and terminated by lysis in a further four days, the patient eventually making a good recovery.

CHART II.

EFFECT OF INJECTION OF BLOOD HAEMOLYZED BY HEAT.

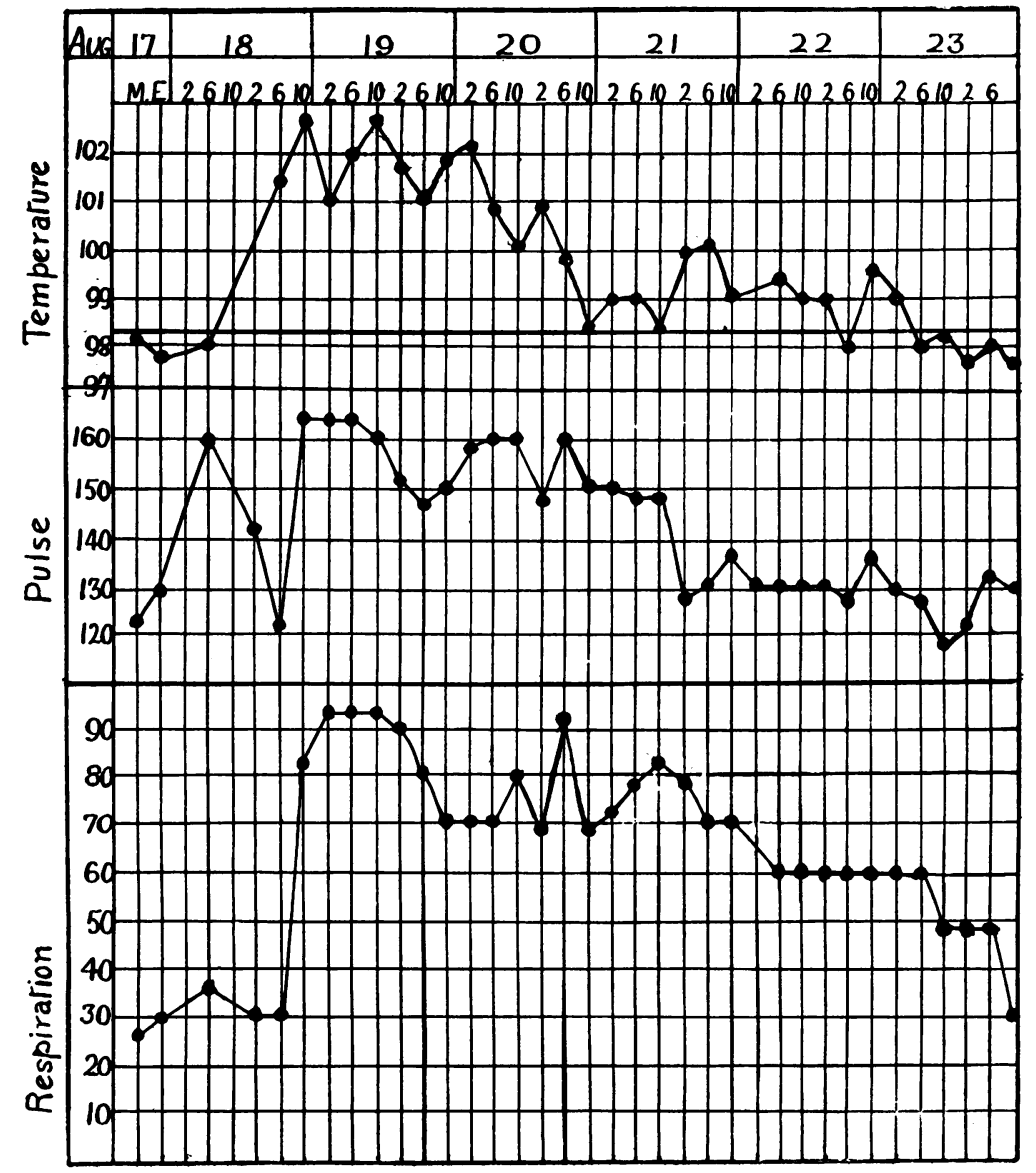


The reaction of this patient was so violent that it was decided not to make any confirmatory experiments. Her behaviour, however, taken in conjunction with the rise of mortality from roughly 20 per cent. in all previous cases to 60 per cent. in 1933, in which year the summer was abnormally hot, and with the death of a patient following accidental exposure to sunlight, suggested that heat was an important factor in causing the condition, and that cooling the patients would have a beneficial effect on the course of the malady.

\section{Effects of cooling.}

In 1934 twelve patients were admitted, and in all cases treatment was directed towards keeping the patients cool. The methods adopted to attain this end were:-

1. The patients were lightly clad, one garment only being worn. They were not covered by bedclothes.

2. Three times a day they were sponged with cold water.

3. An electric fan was placed in the room.

4. The patients, if they liked, were allowed frequently to immerse their hands in a bowl of cold water, as suggested by Rocaz ${ }^{3}$. The majority of them appreciated this greatly.

The results of this treatment were striking. The summer was if anything hotter than that of 1933, but of the twelve cases admitted only one died (8.3 per cent.). The fatal case was that of a patient who contracted an infective enteritis very soon after admission and died in five days. Another patient was apparently cured and discharged a fortnight after admission, in November. However, he relapsed in. January, 1935, and was readmitted. His second attack was the worst example of pink disease ever seen by the author, the teeth falling out and gangrene occurring in both feet and the lower jaw. The patient eventually died in March. In view of the severity of the illness the resistance put up by this patient was remarkable. Table 1 shows the results of this method of treatment.

TABLE 1.

\begin{tabular}{|c|c|c|c|c|}
\hline Case & $\begin{array}{l}\text { AGE IN } \\
\text { MON'THS }\end{array}$ & $\begin{array}{l}\text { DURATION OF } \\
\text { ILLNESS BEFORE } \\
\text { T'REATMENT }\end{array}$ & Result & $\begin{array}{l}\text { DURATION OF } \\
\text { TREATMENT }\end{array}$ \\
\hline A. $\mathbf{M}$. & 22 & 4 months & Cure & 18 days \\
\hline L. M. & 9 & 1 month & Cure & 5 weeks \\
\hline T. G. & 15 & 4 months & Cure & 3 weeks \\
\hline P. $\mathbf{R}$. & 9 & 2 months & Much improved & 6 weeks \\
\hline M. P. & 8 & 1 month & Cure & 3 weeks \\
\hline B. $\mathbf{L}$. & 8 & 1 month & Cure & 4 months* \\
\hline P. P. & 9 & 3 months & Cure & 4 weeks \\
\hline K. P. & 7 & 1 month & $\begin{array}{l}\text { Death from } \\
\text { enteritis }\end{array}$ & 5 days \\
\hline J. M. & 11 & 4 months & Cure & 4 weeks \\
\hline D. C. & 7 & 1 month & Cure & 4 weeks \\
\hline M. M. . & 8 & 2 months & Cure & 6 weeks \\
\hline B. H. & 12 & month & Apparent cure & 2 weeks $\dagger$ \\
\hline
\end{tabular}

* This was the patient in whom a relapse was apparently induced by the injection of heated blood. She was kept in hospital a long time to make sure that no further relapse occurred.

$\dagger$ This patient relapsed and died in March, 1935. 
Discussion.

It is well known that physical agents such as heat and cold can act as toxic substances.

So long ago as 1916 Storm van Leeuwin and van der Made ${ }^{4}$ showed that raising or lowering the temperature of a decerebrate cat above or below the optimum of $38^{\circ} \mathrm{C}$. profoundly influenced the reflex excitability of the nerves. This was greatly diminished by only slightly raising the temperature, whereas, considerable lowering was needed to produce any definite results. Similar results were obtained in the decerebrate frog.

Heymans" studied the effects of raising and lowering the temperature of the blood in the rabbit by interposing a U-tube which could be subjected to varying temperatures in a carotido-jugular anastomosis. By rapidly raising the temperature of the U-tube to $90^{\circ} \mathrm{C}$. the rectal temperature of the animal rose to 39 to $39 \cdot 8^{\circ} \mathrm{C}$. By cooling the anastomosis the temperature fell, and could be again raised on further heating. After the third repetition the animal died in convulsions. But when cooling and heating of the blood was done gradually, rectal temperatures varying between 34 to 32 and 41 to $42^{\circ} \mathrm{C}$. could be produced, and the animal invariably survived. If the rectal temperature was raised to $43^{\circ} \mathrm{C}$., even if as long as three hours was taken to do it, death in convulsions was invariably produced; but if the rectal temperature did not exceed $42 \cdot 2^{\circ}$ C., and the anastomosis was subsequently cooled, the rabbit recovered. Cooling produced similar but much less marked results, mortal hypothermia being estimated at 18 to $20^{\circ} \mathrm{C}$. Heymans considered that the toxic effects observed were due to the direct action of heat alone, and not to the destruction of the blood or to the formation of a chemical substance therein, for if the blood were heated to a temperature of $50^{\circ} \mathrm{C}$. but cooled before its return to the body, no ill effects were observed. However, if the temperature of the blood were raised to 55 to $57^{\circ} \mathrm{C}$., even though the rectal temperature only rose to $39.8^{\circ} \mathrm{C}$., haemolysis occurred and the animal died with haematuria, haemoglobinuria, and parenchymatous nephritis. The heart and lungs were found to be able to stand their temperature being raised to $47^{\circ} \mathrm{C}$. or more without apparent ill effect, so that it was assumed that the cause of death in hyperthermia was due to the effect of heat on the brain. Support for this hypothesis was forthcoming in an experiment in which the brain alone had its temperature increased (that of the trunk being only $35 \cdot 2^{\circ}$ C.), the animal dying in two minutes.

These experiments of Storm van Leeuwin and van der Made, and of Heymans demonstrate that heat even in small amounts acts as a toxic agent, and that the site of this action is in the nervous system. The hypothesis of the latter that it is due to heat alone and not to the formation of a noxious substance in the blood, cannot be accepted as proved, as possibly a thermolabile substance could be formed, which would cease to exist as such when the blood was returned to the body.

The toxic effects of heat, light, and other physical agents on man have been extensively studied by Dukes. Having first observed and reported a case of urticaria caused by light ${ }^{6}$, he collected a further series of cases of this affection which were apparently due to such causes as heat, light, cold, freezing, burns, mechanical irritation and physical and mental exertion ${ }^{7}$. Later, in an important paper ${ }^{8}$ he postulated a condition, akin to allergy, of heat-, effort-, and cold-sensitivity. He points out that heat may be regarded as a waste product, and that as an abnormal rise in the temperature in a given organ is damaging to its health and activity, so 
an abnormal rise of the body temperature in general is damaging to the health and activity of the subject. Under normal conditions there is a remarkably efficient and complex mechanism which provides adequate cooling both of individual organs and of the whole body. Defective heat regulation may give rise ta a variety of disorders affecting the body as a whole, or affecting the function of one of the internal organs, and finally may give rise to abnormal responses in one of the three important cooling surfaces-skin, nasal mucous membrane, and bronchial mucosa-and may cause serious pathological changes in these localities. Examples of general constitutional reactions are afforded by heat prostration and effort syndrome; of those referable to one of the cooling surfaces, thermic coryza, asthma (or more commonly a sense of oppression in the chest associated with cough, often complicated by a superimposed infection producing bronchitis), urticaria and eczema; of those referable to an internal organ, thermic headache or a gastric upset caused by a change of temperature or effort. The outstanding feature of all these disorders is that they can be brought about by heat, effort, or cold, as the case may be, and can be temporarily relieved by the reverse conditions.

Other skin affections observed by this author resulting from sensitivity to physical agents include erythema, itching, angioneurotic oedema, and, if the condition is chronic, desquamation. If to these manifestations be added nasal catarrh, depression, and prostration-all recorded symptoms of heat sensitivity-and the clinical picture so produced be transferred to the age of the first dentition, no one who has seen a typical case would hesitate to diagnose pink disease.

If it is assumed that pink disease is a manifestation of heat sensitivity, this would explain the seasonal incidence in this country. It would also explain the fall during midsummer and the rise at the beginning and end of the hot season, for according to Duke many heat sensitive subjects only react after previous exposure to cold. They become tolerant to heat during the summer, and lose their tolerance during the winter, so that the first warm days of spring produce a reaction. He further describes a type which reacts only after the first cold days of winter. These patients lose their tolerance to heat after a brief exposure to cold, and show symptoms during a subsequent warm spell.

Jeffreys and Ian Wood $^{9}$ in a recent paper reject the theory that pink disease is an abnormal rection to daylight because (i) they have found that patients who are nursed in the open air have a shorter illness than those who are nursed indoors; (ii) graded sun baths sufficient to produce tanning have been shown to be beneficial; and (iii) an analysis of 116 cases failed to show that the greatest number occurred at a time of year when the hours of sunshine were at a maximum. It should be noted that their objections are directed against the suggestion that light, not heat, is the responsible factor. If the disease is due to heat sensitivity it is easy to see why open air treatment is beneficial, while the benefit obtained by graded sun baths may well be due to a process of desensitization to heat; in fact it is directly comparable to the process of desensitization in ordinary chemical allergy, such as the injection of graded doses of pollen extract in hay fever. Lastly, the analysis 
of the seasonal incidence of their cases shows a marked increase at the time when the days are beginning to lengthen after the winter, a fact which tends to support the hypothesis of heat sensitivity rather than to contradict it. Similarly Brown, Courtney, and MacLachlan ${ }^{10}$ found that no cases occurred in the summer or winter months, their seven cases being observed in April, May, June, and October. On the other hand, de Cosmi ${ }^{11}$ found that the greatest incidence in eighty-nine cases was in the first six months of the year, sixty being recorded from January to May. The appearance of the affection during the cold weather may be due to the patients losing their heat tolerance during this time, and the onset may be determined by mistaken efforts to keep the child warm. Once even mild symptoms have developed the cold and clammy hands and feet and the perpetually running nose often cause the mothers to clothe the children warmly and to keep them in bed near a hot fire, which are disastrous measures for a heat-sensitive child. Possibly, too, some cases are due to the opposite condition of cold sensitivity, as is suggested by the following case (not included in this series):-

P.D., girl, aged nineteen months, was seen on December 17, 1935, for lack of vitality and weakness. She was the only child of healthy parents and had been fed on a 'humanized' dried milk, being apparently normal, for the first six months. At nine months she was given a mixed diet, although she never had gravy or meat, as her parents were vegetarians. She had, however, ample green vegetables, eggs, and milk. At six months, in December, she began to get weak and liable to attacks of depression. The hands and feet became red and swollen, and this was more obvious whenever the weather was cold. The hair began to fall out. The appetite remained good, there was no sweating, sleep was normal, and there was no nasal catarrh. The child picked up during the summer and had made good progress until the last few weeks, when there was a recrudescence of the swelling and redness of the hands and feet. The patient was seen on the first day of a period of cold weather, and the hands and feet were very red and swollen. The gait was rather unsteady and the child was fretful. Otherwise no abnormality was detected.

The above is admittedly a doubtful case, and the patient seemed convalescent. But it was difficult to diagnose any other condition than pink disease, and the dependence of the symptoms on a low temperature of the air was marked.

\section{The nature of heat sensitivity in pink disease.}

Although this investigation originated in observing the haemolytic effect of heat, it is obvious that in pink disease there is no haemolysis, as neither haemolytic jaundice, anaemia, or haemoglobinuria occurs, and in several cases a van den Bergh reaction was carried out with negative results. Moreover, the blood of patients with pink disease does not haemolyze at a lower temperature than normal blood. Morey and Michie ${ }^{12}$ found that a patient suffering from a degree of heat sensitivity sufficient to cause urticaria, nausea, vomiting, palpitation, and fainting was much improved by injections 
of histamine. Duke, however, found that sensitive patients react to heat applied to the forearm even when a tourniquet is applied to the upper arm, so that the production of a noxious chemical substance in the blood cannot be responsible. This is the same conclusion that Heymans came to as a result of his animal experiments. In pink disease there is apparently no marked skin sensitivity to heated blood, and although in the case reported in this paper a profound reaction followed the subcutaneous injection of heated blood, this was probably only a manifestation of hypersensitivity to serum injections in general, which according to Duke is characteristic of all heat-sensitive persons. It seems, therefore, most probable that all the tissues are sensitive to the direct action of heat, and that symptoms are more easily produced in the nervous system and in the cooling apparatus of the body than elsewhere.

\section{The infective theory of pink disease.}

The majority of observers consider pink disease to be due to infection by a specific micro-organism. The almost invariable association of the condition with catarrh of the respiratory tract, its appearance in this country only during the last fifteen years, its greater prevalence in some districts than others, and the liability to death from fulminating secondary respiratory infections are all suggestive of a specific infection. Nevertheless, many of these facts are explicable on other grounds. Cases may well have occurred previously and not have been diagnosed; the increase in incidence is synchronous with the increase in the popularity of sunbathing, and this appears to vary in different localities at different times, so that a condition of acquired heat sensitivity might assume the appearance of an epidemic. The liability to respiratory catarrh and to bronchopneumonia are just as likely to be allergic phenomena as manifestations of a primary infection.

On the other hand the following observations are highly suggestive that the condition is not due to infection:-

1. On the whole the illness is afebrile. The temperature tends to be unstable, with occasional rises above the normal line, but not to any degree. Any pronounced or prolonged pyrexia generally indicates the occurrence of a secondary infection.

2. The blood sedimentation rate was within normal limits in thirteen out of twenty-one cases. This fact almost precludes any active infection. It is suggested that an increased sedimentation rate points to a secondary infection.

3. Leucocytosis is not constant and the proportion of the polymorphs to mononuclear cells is not necessarily increased. The white cells per c.mm. were increased above the number of 12,000 in only sixteen of thirty-five patients.

4. No specific micro-organism has been isolated, and the bacteriological findings from the respiratory tract present no constant features. 
Table 2 shows the white cell counts in thirty-four cases and the sedimentation rates in twenty-one.

TABLE 2.

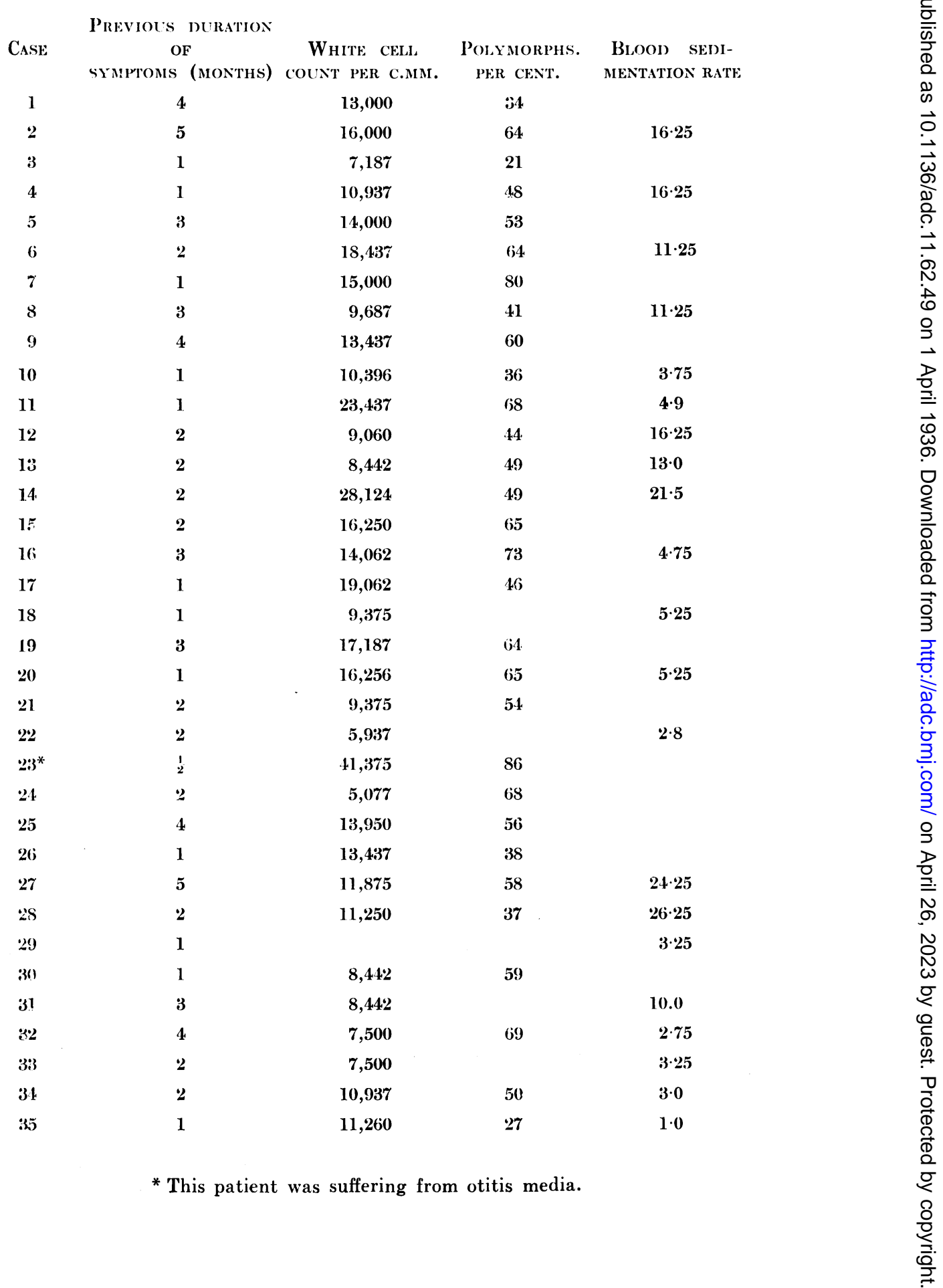


Thanks are due to colleagues in the pathological department, Dr. W. W. Mackarell and Dr. Mary Sharpe, for their help and advice, to Mr. C. J. Bond for his valuable assistance and advice in the experiments on haemolysis, and to successive house physicians who have so willingly carried out the routine work in connection with these cases.

\section{Summary.}

1. Pink disease occurs most frequently in the spring and late summer in this country.

2. One patient, previously reported, showed toxic symptoms on being placed in the sunlight, while another was accidentally exposed to sunlight through glass and died on the same day.

3. In the hot summer of 1933 , six out of ten patients died, in spite of their being protected from the rays of the ultra-violet end of the spectrum.

4. In 1934, twelve patients were treated by cooling measures-hydrotherapy and light clothing. Only one case terminated fatally, death occurring from a ward infection five days after admission.

5. Blood in glass tubes exposed to sunlight was haemolyzed. This was proved to be due to heat rays. Blood haemolyzed by heat was shown to be highly toxic to a patient with pink disease when injected subcutaneously.

6. Adult patients with sensitivity to heat, effort, and cold show abnormal responses to these agents in the three important cooling surfaces of the body-skin, nasal and bronchial mucous membranes. All the symptoms described in adult physical allergy are present in pink disease.

\section{Conclusions.}

1. Sensitivity to physical agents, especially to heat, plays an important part in the production of the symptoms of pink disease.

2. Cooling the patients by light clothing, hydrotherapy, and keeping them out of direct sunlight greatly reduces the mortality and shortens the course of the disease.

\section{REFERENCES.}

i. Braithwaite, J. V., Arch. Dis. Childh., London, 1933, VIII, 1.

¿. Clare, T. C., Brit. Med. Journ., London, 1925, ii, 640.

̋. Rocaz, Ch., Pink Disease, transl. by Jeffreys Wood, London, 1933, 37. 
4. Storm van Leeuwin, W., \& van der Made, M., Pflüger's Arch. f. d. ges. Physiol., Berlin, 1916, CLXV, 37.

5. Heymans, .J. F., Arch. internat. de pharmacodyn. et de thérapie, Brussels, $1919, \times X V, 1$.

6. Duke, W. W., J. Am. Med. Ass., Chicago, 1923, LXXX, 1835.

¡. Duke, W. W., ibid., 1924, LXXXIII, 3.

8. Duke, W. W., Arch. Int. Med., Chicago, 1930, XLV, 206.

9. Wood, J., \& Wood, I., Brit. Med. J., London, 1935, ii, 52\%.

10. Brown, A., Courtney, A. M., \& MacLachlan, I. F., Arch. Pediat., New York, 1921, XXXVIII, 609.

11. De Cosmi, S., quoted by Rocaz, loc cit.

12. Morey, J. B., \& Michie, T. C., Proc. Staff Meet. Mayo Clin., Rochester, 1930, $\mathrm{V}, 74$. 